\title{
La violencia contra las mujeres con discapacidad en tiempos de COVID-19 y experiencias grupales de sororidad online
}

\author{
Violence against women with disabilities in times of COVID-19 and \\ experiences of sisterhood online groups
}

\section{Palabras clave}

Violencia de género, mujeres con discapacidad, COVID-19, buenas prácticas, sororidad.

\section{Keywords}

Violence against women, women with disabilities, COVID-19, good practices, sisterhood.

\author{
Esther Castellanos-Torres \\ $<$ esthercastellanostorres@gmail. \\ com> \\ Fundación CERMI Mujeres. España

\section{Isabel Caballero Pérez} \\ <coordinacion@fundacioncermi \\ mujeres.es> \\ Fundación CERMI Mujeres. España
}

\section{Las gafas violetas para entender el impacto del COVID-19 en la vida de las mujeres}

Desde que el coronavirus irrumpiera en nuestra sociedad desde hace ya ocho meses, ha quedado constatado que la pandemia y las medidas institucionales derivadas para su contención han tenido y tienen impactos diferenciales sobre la vida de mujeres y hombres. Además, existe evidencia de llamamientos (Wenham et al., 2020: 846-848) e informes realizados desde organismos internacionales como ONU Mujeres (2020a, 2020b) en los que se insta a que las respuestas que se den desde los Estados para mitigar los efectos del COVID-19 tomen en consideración el enfoque de género.

Así, el propio Ministerio de Igualdad publica La perspectiva de género, esencial en la respuesta a la COVID-19, informe realizado por el Instituto de la Mujer y para la lgualdad de Oportunidades en el que se hace eco de que las consecuencias económicas, sociales y familiares son distintas en mujeres y hombres. Suma evidencia del mayor impacto en las mujeres por los aspectos vinculados directamente con las desigualdades estructurales de género y que tiene que ver con la feminización del sector de trabajo sanitario y de servicios esenciales, con la falta de corresponsabilidad aun

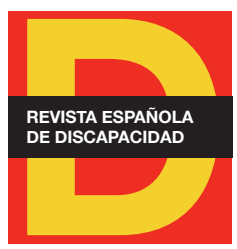

Para citar:

Castellanos-Torres, E. y Caballero, I. (2020). "La violencia contra las mujeres con discapacidad en tiempos de COVID-19 y experiencias grupales de sororidad online". Revista Española de Discapacidad, 8(2), pp. 211-221.

Doi: $<$ https://doi.org/10.5569/23405104.08.02.10> 
en el entorno privado y doméstico. Según datos del Eurostat (European Commission, 2019), en España, un $95 \%$ de las mujeres se involucran en el cuidado de sus hijos e hijas diariamente, frente a un $68 \%$ de los hombres. Otro de los aspectos que sitúa a las mujeres en una posición de desigualdad, acentuando la brecha ya existente por la crisis, es la precariedad laboral y la feminización de la pobreza.

Como señala Lucía Gallego (2020) haciéndose eco de Sophie Cousins (2020) tampoco podemos olvidar otros factores que están influyendo negativamente sobre las mujeres en la era COVID-19, como son el aumento de la violencia de género y la dificultad de acceso a servicios de salud en todo el mundo, la mayor probabilidad de perder el empleo, o el aumento de las tareas de cuidados no remunerados y efectos en la salud general y mental de las mujeres, lo que afecta de manera dramática la calidad de vida.

Diversas organizaciones feministas alertaron del aumento de la violencia machista durante la situación de confinamiento, en que las mujeres víctimas de violencia de género deben convivir con su agresor y sus movimientos están limitados. En el caso de las mujeres con discapacidad, están más expuestas, ya que existe una mayor dependencia de las personas cuidadoras (Castellanos-Torres et al., 2020: 420).

Así, en el propio informe referido anteriormente, se señala que los datos de llamadas y consultas online al teléfono 016 durante el periodo de confinamiento, difundidos por la Delegación de Gobierno contra la Violencia de Género (DGVG), son una manifestación de este impacto. En el caso de las llamadas, durante la primera quincena del mes de abril con respecto a la primera quincena del mes de marzo, en 2020, se produjo un incremento del $48 \%$, que asciende hasta el 733,3\% en el caso de las consultas online. Comparando el periodo del 14 de marzo al 15 de abril de 2020 con respecto al mismo en 2019, los incrementos han sido del $31 \%$ en el número de llamadas y del 443,5\% en el de consultas online. Por esta razón, el Ministerio de Igualdad puso en marcha un Plan de contingencia contra la violencia de género ${ }^{1}$ y otro Plan para garantizar los derechos de las víctimas de explotación sexual y en contexto de prostitución, con dificultades para acceder al sistema público de salud. Podemos encontrar el contenido detallado de las medidas institucionales a nivel nacional e internacional en la revista Gaceta Sanitaria (Ruiz-Pérez y Pastor-Moreno, 2020).

En este contexto cabe preguntarnos y reflexionar sobre qué estaba pasando con las mujeres con discapacidad, una vez más. ¿Se pondría de manifiesto la falta de accesibilidad de los recursos de atención a la violencia de género, tal y como advierte Fundación CERMI Mujeres²?

\section{La violencia contra las mujeres con discapacidad: una pandemia previa a la pandemia}

En palabras de Phumzile Mlambo-Ngcuka, directora ejecutiva de ONU Mujeres, esta nueva realidad provocada por el coronavirus ha puesto de manifiesto "cómo aumenta otra pandemia en la sombra: la violencia

\footnotetext{
1. Real Decreto-ley 12/2020, de 31 de marzo, de medidas urgentes en materia de protección y asistencia a las víctimas de violencia de género. Recuperado de https://www.boe.es/eli/es/rdl/2020/03/31/12.

2. Nota de prensa CERMI Mujeres pide que las medidas para reforzar la protección a víctimas de violencia machista durante el confinamiento tengan en cuenta la variable de discapacidad (3 de marzo 2020). Recuperado de https://www.cermi.es/es/actualidad/noticias/cermi-mujeres-pideque-las-medidas-para-reforzar-la-protecci\%C3\%B3n-v\%C3\%ADctimas-de.
} 
contra las mujeres" (ONU Mujeres, 6 de abril de 2020). En este punto, tendríamos que preguntarnos qué ocurre cuando las violencias y el confinamiento se dan en mujeres con discapacidad. Sin duda, es clave la mirada interseccional (Crenshaw, 1989) de las mujeres, ya que es necesario ponerse unas gafas no solamente feministas para analizar el origen estructural de la violencia de género, sino que es preciso hacerlo con un doble o triple aumento (Castellanos-Torres, 31 de mayo de 2020). Además, la interseccionalidad es una teoría que se ha mostrado de manera notoria en esta crisis, ya que los efectos de la misma en el caso de una mujer, mayor, migrante, residente en el mundo rural y pobre están siendo mucho más devastadores, precisamente por los ejes de opresión que potencian la discriminación (CERMI, 2020: 76). Por lo tanto, es pertinente incidir en esta mirada, sobre todo si somos conscientes de que casi el $80 \%$ de las mujeres con discapacidad sufren violencia y tienen cuatro veces más riesgo de sufrir violencia sexual que el resto de las mujeres (Valenciano, 2004). El proyecto "Stop the Violence” descubrió que la violencia se intensificó en frecuencia, extensión y naturaleza cuando el género y la discapacidad se cruzan (Dowse et al., 2013).

Pero además, en marzo de 2020 Women Enabled International (WEI) identificó una brecha en las respuestas globales iniciales al COVID-19, ya que institucionalmente se estaba discutiendo cómo incluir a personas con discapacidad en dichas respuestas, pero pocas de ellas estaban considerando las experiencias únicas de las mujeres con discapacidad. A su vez, la WEI se hizo eco de las organizaciones de mujeres con discapacidad que estaban angustiadas por la situación en sus países y tenían preocupaciones sobre las mujeres de este sector de población, especialmente en torno a la violencia, al acceso a bienes y servicios de salud y a la satisfacción de necesidades básicas.

En este sentido, se pone de manifiesto que esta crisis sigue sin asegurar el enfoque de género en la respuesta que se está ofreciendo a las personas con discapacidad. Ésta debe considerar de manera diferenciada las necesidades particulares de las mujeres y niñas en el nivel general, pero también las diferentes necesidades que pueden plantear dentro de una misma discapacidad. No se trata de prestarles a ellas mayor apoyo, sino de garantizar que todas las personas con discapacidad que lo precisen sean beneficiadas por la asistencia sin discriminación por sexo, a la vez que tener presente las necesidades específicas de mujeres y niñas con discapacidad. Tampoco se han contemplado acciones que protejan a las madres y cuidadoras, para que esta emergencia no impacte de forma desmedida en las mujeres, quienes mayoritariamente se responsabilizan de asumir los cuidados en España (CERMI, 2020: 44)

En su informe, WEI (2020) refiere que las mujeres y niñas con discapacidad sufren violencia por parte de sus parejas y familiares al menos dos o tres veces más que otras mujeres, y el confinamiento domiciliario y otros momentos de obligatoriedad o aislamiento recomendado, hace que las mujeres tengan menos posibilidades de escapar de la violencia, sobre todo si no pueden contar con sus apoyos habituales. Además, la disminución del acceso a los servicios formales de apoyo significa que las mujeres dependerán más de métodos informales de apoyo, lo que puede exponer aún más a la violencia, la explotación y el abuso. Sin olvidar la situación de las mujeres con discapacidad institucionalizadas que corren un mayor riesgo de sufrir violencia debido a su aislamiento, que aumenta cuando no se permite la entrada de familiares u otras visitas.

En esta misma línea el informe del CERMI (2020: 30) incide en que el confinamiento puede ser un espacio propicio para la violencia machista, que en el caso de las mujeres con discapacidad tienen que afrontar el confinamiento sin apenas apoyos, con dificultades derivadas de la falta de suministros de diferentes servicios, o falta de ayudas en el seguimiento escolar de sus hijas e hijos, entre otros. 
Pero además, tal y como apunta Miguel Lorente-Acosta (2020), el confinamiento, por su parte, crea las condiciones idóneas para que los elementos de la violencia de género se potencien: aísla aún más a las mujeres, aumenta el control por medio de la clausura formal en el hogar, facilita la impunidad al aumentar las barreras para que las mujeres puedan salir de la violencia y crea un contexto que facilita el uso de cualquiera de sus formas ante el más mínimo estímulo.

Además, y según los últimos datos publicados de la Macroencuesta de Violencia contra la Mujer 2019 por la Delegación del Gobierno contra la Violencia de Género (DGVG, 2020) el 17,5\% de las mujeres con discapacidad que han sufrido "violencia física, sexual, emocional o han sentido miedo" de sus parejas (VFSEM) de alguna pareja, afirman que su discapacidad es consecuencia de la violencia ejercida sobre ellas por sus parejas. Un 17,4\% de las mujeres que tienen una discapacidad acreditada ha sufrido violencia física de alguna pareja frente al $11 \%$ de las mujeres sin discapacidad. Un $23,4 \%$ tiene una discapacidad como consecuencia de algún episodio de violencia física o sexual.

Por lo tanto, en un periodo de reclusión impuesta hay que poner toda la vigilancia y los recursos, en clave de inclusión, para que las mujeres con discapacidad se sientan protegidas y, sobre todo, vivas a pesar del confinamiento. Desde un planteamiento feminista también hay que mirar a las madres y cuidadoras, responsables en gran medida de la atención de las personas con discapacidad, para las que no se han contemplado acciones que las protejan y las cuiden en un momento tan crítico (CERMI, 2020: 45)

Llegadas a este punto, Ana Peláez (2020), vicepresidenta ejecutiva de la Fundación CERMI Mujeres (FCM) advierte de la situación y del mayor impacto en las mujeres con discapacidad y expone un decálogo de recomendaciones para que las entidades del sector de la discapacidad puedan reforzar el apoyo y seguimiento a las mujeres y niñas con discapacidad en estas circunstancias ${ }^{3}$. Así, desde la FCM se articula una respuesta interseccional a la violencia contra las mujeres en tiempos de COVID-19.

Una de las primeras decisiones adoptadas durante el mes de marzo, fue la elaboración de la Guía de orientaciones prácticas de denuncia de la violencia de género sobre mujeres y niñas con discapacidad en la situación de emergencia por el coronavirus (FCM, 2020), como complemento a la guía publicada por el Ministerio de Igualdad (2020) en las mismas fechas. Se necesitaba asegurar la plena accesibilidad de las vías de denuncia y acceso a la información en este ámbito, algo que se garantizó con la puesta a disposición de todas las mujeres con discapacidad de los contactos de las y los profesionales que desde los distintos territorios y organizaciones sectoriales del movimiento CERMI permanecían en activo atendiendo a las mujeres que lo demandasen, así como la creación de una dirección de correo electrónico donde pudieran dirigir sus solicitudes y denuncias en materia de violencia de género. El servicio de atención jurídica de la FCM era la instancia encargada de recibir las consultas y darles respuesta.

3. Cermi semanal n 384. Recuperado de http://semanal.cermi.es/noticia/mujeres-discapacidad-coronavirus-ana-pelaez-cermi-mujeres.aspx. 


\section{Tejiendo sororidad frente a la crisis derivada del COVID-19, la buena práctica de "No estás sola" de la Fundación CERMI Mujeres}

Alertadas por las dificultades extraordinarias que muchas de las mujeres con discapacidad debían enfrentar en un contexto tan excepcional a la hora de denunciar situaciones de violencia o de acudir a recursos especializados durante el período de confinamiento decretado a través del estado de alarma en el país, la FCM decidió poner en marcha una iniciativa cercana a la metodología de los grupos de ayuda mutua y de intervención grupal con mujeres víctimas de violencia de género. En este sentido, dicha metodología se ha mostrado eficaz para iniciar el camino hacia el empoderamiento, ya que los espacios grupales ayudan a las mujeres a conectarse con otras y a compartir experiencias vitales. Hay certeza de que los grupos de mujeres son una alternativa preventiva para la promoción de la salud. El intercambio de percepciones, ideas, sentimientos y proyectos, a algunas mujeres les permite salir del aislamiento y participar en un espacio de escucha, donde compartir sus experiencias, sentirse comprendidas y tomar conciencia de su situación (Migallón y Gálvez, 1999). Y, sobre todo, porque trabajar en grupos nos hace ser conscientes y darnos cuenta de que muchos de los sentimientos, experiencias y problemáticas que hemos sentido o nos han hecho sentir como cuestiones individuales e intrapsíquicas, son comunes a muchas mujeres, marcadas por el género y la cultura (Nogueiras et al., 2005) y favorece la exposición a modelos alternativos de pensar, sentir y actuar (Caballero et al., 2012). En este sentido, la iniciativa buscaba poner en común a todas las mujeres con discapacidad y mujeres cuidadoras de personas con discapacidad.

Sin embargo, el principal hándicap que existía para materializar esta iniciativa venía derivado de la herramienta de comunicación que debía ser utilizada para poner en contacto a decenas de mujeres con discapacidad no solo de España sino también de la comunidad latinoamericana, y es que el uso de las nuevas tecnologías se presentaba como una oportunidad, pero sin olvidar que nunca antes la FCM había utilizado instrumentos telemáticos de comunicación, dada las evidencias, entre otras (Vicente et al., 2005; Gomiz, 2016; Fundación Vodafone, 2013) de la brecha digital, junto con la convicción de que la inaccesibilidad y falta de alfabetización digital dificultarían su uso en actividades que involucrasen a las mujeres con discapacidad.

Sin embargo, la respuesta fue -y sigue siendo- abrumadora ${ }^{4}$. La brecha digital parecía un obstáculo que se diluía frente a las ganas y la necesidad de contacto, puesta en común de experiencias, ruptura del aislamiento e intercambio de información. Como una suerte de grupo virtual de apoyo mutuo, las mujeres con discapacidad fueron tejiendo en esos meses de primavera y de comienzo del verano estrategias de acompañamiento y supervivencia en un contexto a todas luces hostil. Elena Briongos, ${ }^{5}$ afirmaba "este dichoso coronavirus nos ha cambiado la vida y la forma de relacionarnos, voy a intentar recoger lo bueno de aquellas reuniones de mesa camilla, aprovechando que escucháis desde vuestras casas y yo hablo desde la mía". La mesa de camilla como metáfora del grupo de mujeres que intercambian sus voces a través de las nuevas tecnologías, hace que éstas se conviertan en aliadas a través de todos los apoyos necesarios, garantizando su accesibilidad a todas las mujeres según su discapacidad.

4. Ha sorprendido la importante movilización de mujeres con discapacidad en un periodo que amenazaba con convertirse en un tiempo de desconexión.

5. Es la presidenta de Salud Mental Castilla y León y fueron pronunciadas en el II Webinario No estás sola "La salud de las mujeres con discapacidad en el contexto de la pandemia" el 6 de mayo de 2020 
Este acompañamiento de mujeres y niñas con discapacidad, madres, profesionales y cuidadoras fue denominado "No estás sola" articulándose a través de sesiones virtuales semanales sobre diferentes temáticas de interés para las mujeres del movimiento CERMI y para otras mujeres con discapacidad de la comunidad latinoamericana.

Por esta vía llegaba información de lo que estaba sucediendo en centros residenciales de personas mayores y de personas con discapacidad donde el COVID-19 se ensañaba con toda su crueldad. Los déficits del sistema residencial español y la precariedad de las políticas de cuidados salieron a la luz, siendo las principales damnificadas, una vez más, las mujeres con discapacidad. Siguiendo directrices no escritas, los hospitales rechazaban en sus unidades de cuidados intensivos a personas con discapacidad, reeditando de esta manera viejos paradigmas que se creían ya superados - el paradigma de la prescindencia (Palacios, 2008), por ejemplo6 - que emergía sin prejuicios en esta nueva cotidianidad y se aplicaba en aras del bien común?

Las mujeres cuidadoras de personas con discapacidad, muchas de ellas también con discapacidad, se convirtieron, asimismo, en grupo meta de la iniciativa, conscientes del importante papel que han venido desarrollando en este periodo y en reconocimiento a la labor que desempeñan en un estado de bienestar muy reducido, en el que su contribución no monetarizada permite a este país seguir funcionando. En palabras de $\mathrm{M}^{\mathrm{a}}$ Ángeles Durán (2010) "la calidad de vida y el bienestar de un país están estrechamente ligados a la disponibilidad de cuidado suficiente y de buena calidad. Ninguna otra actividad de la economía española consume tanto trabajo o produce tanto bienestar como el cuidado".

Asimismo, en estos encuentros las niñas y jóvenes con discapacidad denunciaron su exclusión de las clases de educación a distancia ensayadas con toda precipitación en los colegios e institutos de todo el país. Las metodologías utilizadas no eran, en la mayor parte de los casos, accesibles ni tampoco los materiales que se distribuían entre el alumnado para que pudieran seguir los contenidos del currículo escolar desde sus casas. La falta de apoyo ha sido la tónica general en este periodo, demostrando las carencias del sistema público de educación en España y su falta de inclusividad².

También se puso de manifiesto cómo temas que vulneran los derechos humanos, tales como la explotación sexual y reproductiva de las mujeres, o la realidad de las mujeres adultas mayores, o los entornos rurales,

6. Un rasgo esencial del modelo de prescindencia es la consideración de que las personas con discapacidad no tienen nada que aportar a la comunidad. La idea de que la vida de una persona con discapacidad no merece la pena ser vivida, sumada a la creencia acerca de su condición de carga - para los padres, madres o para el resto de la comunidad- origina que la solución adoptada sea prescindir de estas personas, mediante el recurso a prácticas eugenésicas.

7. Rosa, una mujer contagiada con discapacidad intelectual: "En el hospital nos dijeron que no entraría en la UCl" eldiario.es 12 de abril de 2020 : https://www.eldiario.es/sociedad/denuncia-uci-discapacidad-intelectual-madrid_1_2263132.html.

8. A este respecto la Fundación Secretariado Gitano, CERMI y Save the Children plantean a los poderes públicos tres medidas clave para revertir la situación antes del 2030. En este sentido, llaman a una reforma de las leyes educativas LOE-LOMCE que reconozca el derecho a la educación inclusiva y recoja medidas concretas de admisión, control y recursos contra cualquier discriminación o trato desigual injustificado, incluido el acoso, en el entorno escolar.

Reclaman también un plan estatal de inclusión educativa con calendario, objetivos, indicadores y presupuesto acordados con las comunidades autónomas para alcanzar un sistema de enseñanza equitativo antes de 2030, así como una nueva Ley integral de igualdad de trato y lucha contra la discriminación que reconozca, prohíba y sancione la exclusión o desigualdad escolares como una forma de discriminación. Fuente: Alianza cívica por la educación inclusiva (2 de julio de 2020) Recuperado de https://www.cermi.es/es/actualidad/noticias/alianza-c\%C3\%ADvica-por-laeducaci\%C3\%B3n-inclusiva. 
son cuestiones que deben ser analizadas, debatidas y resueltas a la luz de un enfoque de doble vía ${ }^{9}$ (UNFPA, 2019) que combine, al menos, la perspectiva de género y la de discapacidad.

En definitiva, estos grupos virtuales han resultado ser herramientas muy útiles en un proceso de la globalización de inquietudes, necesidades e intereses comunes de las mujeres con discapacidad y de poner en marcha una política feminista desde la sororidad. Como señala la antropóloga feminista Marcela Lagarde (2005) la sororidad debe entenderse como una política de la democracia feminista, como una política social y como una forma de relación entre mujeres, que tiene como premisa necesaria el propio feminismo. A su vez, es una experiencia que conduce a la búsqueda de relaciones positivas y la alianza existencial y política, cuerpo a cuerpo, subjetividad a subjetividad con otras mujeres, para contribuir con acciones específicas a la eliminación social de todas las formas de opresión y al apoyo mutuo para lograr el poderío genérico de todas y el empoderamiento vital de cada mujer (Lagarde, 2012: 543).

Pero, al mismo tiempo, la sororidad reivindicada por las mujeres con discapacidad se encuentra impregnada de pensamiento crítico, donde el compromiso compartido no se centra solamente en la lucha contra el patriarcado, sino en la toma de conciencia y en la renuncia del ejercicio del poder de clase, de raza, del capacitismo ${ }^{10}$ (Campbell, 2001) de unas mujeres sobre otras. Esto es poner en práctica el modelo de sisterhood señalado por Bell Hooks (2000/2017), donde se entrecruzan necesariamente diversos ejes de opresión y donde se puede lograr la autorrealización y el éxito sin establecer relaciones de dominio las unas sobre las otras. Así, el reconocimiento de la diversidad entre las mujeres y de la especificidad de cada una es el punto de partida de la sororidad (Lagarde, 2013: 65).

\section{Lecciones aprendidas: sobre la necesidad de implementar iniciativas encaminadas a fomentar el conocimiento de los propios derechos}

Las lecciones aprendidas de este proceso nos permiten evidenciar que el enfoque interseccional subyace en las estrategias emancipatorias empleadas por las mujeres con discapacidad a partir del modelo de la ayuda mutua vehiculizadas a través de la formidable interconexión que producen las redes sociales, generando de esta manera un nuevo espacio global feminista más internacionalista, más diverso, plural e inclusivo. Esto ha permitido que se hayan podido abordar cuestiones clave que se concebían ajenas a las mujeres con discapacidad, al haber estado éstas muy centradas en las luchas contra la violencia y la promoción de la autonomía. Estos espacios plurales interseccionales han facilitado que el debate se haya abierto a ciertas cuestiones que hasta fechas bien recientes habían permanecido ausentes en la agenda política de las muje-

\footnotetext{
9. Este "enfoque de doble vía" implica que, en algunos casos, también son necesarias acciones afirmativas para atender las necesidades de las personas con discapacidad tomando en cuenta el contexto y el ámbito de intervención. Por ejemplo, aunque algunas estrategias de desarrollo tengan en cuenta de manera general los factores de género y discapacidad en el ámbito de la educación, la salud o la prevención de la violencia, es posible que para asegurar la participación plena de las mujeres con discapacidad, se requieran acciones adicionales tales como la entrega de información en formatos adaptados y accesibles, acciones de apoyo específico en el transporte o la infraestructura así como la participación de intérpretes, mediadores o tecnologías específicas para que todas las personas tengan iguales oportunidades.

10. El capacitismo es una red de creencias, procesos y prácticas que produce un tipo particular de yo y cuerpo (el estándar corpóreo) que se proyecta como perfecto, típico de la especie y, por lo tanto, esencial y plenamente humano. Entonces, la discapacidad se presenta como un estado disminuido del ser humano.
} 
res con discapacidad. Se ha otorgado visibilidad a otras demandas expresadas por mujeres de este sector de población que no solían participar en el movimiento social de la discapacidad, al no sentirse del todo o en nada representadas. Al mismo tiempo se han establecido alianzas con otros movimientos de mujeres, abriéndose así una caja de pandora llena de realidades diversas, pero también de intereses comunes, reivindicaciones y daños compartidos.

También se ha constatado la necesidad de promover acciones encaminadas al empoderamiento político de las mujeres a través del conocimiento de los propios derechos, por un lado, y de la detección de las falencias del sistema legal a la hora de garantizar y hacer efectivos los derechos humanos de las mujeres con discapacidad, por otro.

Esto ha tenido como fruto la creación de un Aula de Derechos Humanos donde las mujeres con discapacidad adquieren y generan conocimiento en torno a los instrumentos jurídicos internacionales de derechos humanos clave en este ámbito: la Convención sobre los Derechos de las Personas con Discapacidad, la Convención para la Eliminación de todas las Formas de Discriminación contra la Mujer de Naciones Unidas o el Convenio del Consejo de Europa sobre prevención y lucha contra la violencia contra las mujeres y la violencia doméstica. También este Aula permite acercarse con una mirada interseccional a los 17 Objetivos de Desarrollo Sostenible (ODS), adoptados por la Asamblea General de Naciones Unidas o la Declaración y Plataforma de Acción de Beijing de 1995.

Así ha sido cómo el aislamiento y la desinformación sufridas por muchas mujeres con discapacidad en las primeras fases del confinamiento fueron mitigados con la creación de un espacio propio, de ayuda mutua, donde cada cual puso a disposición del grupo sus conocimientos, sus experiencias y su apoyo para transitar juntas un periodo tan excepcional.

Como cierre y siguiendo a Marcela Lagarde, lo que vivimos hoy, aquí, tiene que ver con lo que viven otras mujeres en sus mundos. Las diversas somos semejantes. Hay conexiones sociales y culturales invisibles entre unas y otras y se condensan procesos culturales que se intersectan sin que nos demos cuenta. Si algo destaca en las feministas y en otras mujeres es la capacidad de pensar y actuar sobre su vida y su mundo, desde un tiempo y un lugar, situadas como mujeres en una genealogía política, con una ubicación de género trastocadora (Lagarde, 2013: 87). 
Caballero, I. et al. (2012). Apoyo psicosocial y prevención de la violencia de género en mujeres con discapacidad. Barcelona: Obra Social "la Caixa”. Recuperado de https://bit.ly/36y1lHG.

Campbell, F. K. (2001). "Inciting Legal Fictions: Disability's Date with Ontology and the Ableist Body of the Law". Griffith Law Review, 10(1), pp. 42-61. Recuperado de https://bit.ly/2VsxcDv.

Castellanos-Torres, E. (31 mayo, 2020). Una mirada interseccional de la violencia contra las mujeres con gafas de doble o triple aumento [Artículo en el blog de invitad@s de Gaceta Sanitaria]. Recuperado de https://bit. ly/39GJfFL.

Castellanos-Torres, E. et al. (2020). "COVID-19 en clave de género [COVID-19 from a gender perspective]”. Gaceta sanitaria, 34(5), pp. 419-421. DOI: https://doi.org/10.1016/j.gaceta.2020.04.007.

CERMI (2020). El impacto de la pandemia de la COVI-19 en los derechos humanos de la personas con discapacidad en España. Colección Convención ONU, № 26. Madrid: Ediciones Cinca. Recuperado de https://bit. ly/3mA5XCR.

Cousins, S. (2020). "COVID-19 has "devastating" effect on women and girls". The Lancet, 396, pp. 301-302. Recuperado de https://bit.ly/3msQQuW.

Crenshaw, K. (1989). "Demarginalizing the Intersection of Race and Sex: A Black Feminist Critique of Antidiscrimination Doctrine, Feminist Theory and Antiracist Politics". University of Chicago Legal Forum, 1, pp. 139-167.

Delegación del Gobierno contra la Violencia de Género, DGVG (2020) Macroencuesta de Violencia contra la Mujer 2019. Ministerio de Igualdad. Recuperado de https://bit.ly/3lwLWvQ.

Dowse, L. et al. (2013). Stop the Violence: Addressing Violence Against Women and Girls with Disabilities in Australia: Background paper. Recuperado de https://bit.ly/36tLjyL.

Durán, M. A. (2010). "Ten Good Reasons for Measuring Unremunerated Work in Health Care”. En VV.AA., The Insivible Economy and Gender Inequalities. The Importance of Measuring and Valuing Unpaid Work (pp. 135136). Washington: Pan American Health Organization (PAHO).

European Commission (2019). The life of women and men in Europe. A statistical portrait. Bruselas: Office for Official Publications of the European Communities. Recuperado de https://bit.ly/2VqoHcb.

Fundacion CERMI Mujeres, FCM (2020). Orientaciones prácticas de denuncia de la violencia de género sobre mujeres y niñas con discapacidad en la situación de emergencia por el coronavirus. Recuperado de https://bit. ly/3o9hLfQ.

Fundación Vodafone (2013). Acceso y uso de las TIC por las personas con discapacidad. Recuperado de https:// bit.ly/36yJYa1.

Gallego, L. (19 agosto, 2020). "Sexo, género y COVID-19: de la negación a la tergiversación de las evidencias" [Artículo en el blog de invitad@s de Gaceta Sanitaria]. Recuperado de https://bit.ly/39l3NgK.

Gomiz, M. P. (2016). "TIC y mujeres con discapacidad una ventana al mundo". Jóvenes e Identidades. Revista de Estudios de Juventud, 111, pp. 119-140. Recuperado de https://bit.ly/3g1HP9K. 
Hooks, B. (2017). El feminismo es para todo el mundo. Traficantes de sueños (Edición original publicada en 2000). Recuperado de https://bit.ly/39AUyis.

Instituto de la Mujer y para la Igualdad de Oportunidades (2020). La perspectiva de género, esencial en la respuesta a la COVID-19. Madrid: Ministerio de Igualdad. Recuperado de https://bit.ly/36wNfGB.

Lagarde, M (2005). Para mis socias de la vida: Claves feministas para el poderío y la autonomía de las mujeres. Colección Cuadernos Inacabados N 48. Horas y Horas.

Lagarde, M. (2012). El feminismo en mi vida: Hitos, claves y topías. México: Instituto de las Mujeres del Distrito Federal. Recuperado de https://bit.ly/3g41NAW.

Lagarde, M. (2013). “Metodologías feministas para la formación de mujeres lideresas”. En M. P. Castañeda (comp.), Perspectivas feministas para fortalecer los liderazgos de mujeres jóvenes (pp. 23-89). México D. F.: Centro de Investigaciones Interdisciplinarias en Ciencias y Humanidades de la Universidad Nacional Autónoma de México. Recuperado de https://bit.ly/3qnAO88.

Lorente-Acosta, M. (2020). "Violencia de género en tiempos de pandemia y confinamiento". Revista española de Medicina Legal, 46(3), pp. 139-145. Recuperado de https://bit.ly/3qm3zlt.

Migallón, P. y Gálvez, B. (1999). Los grupos de mujeres, metodología y contenido para el trabajo grupal de la autoestima. Madrid: Instituto de la Mujer. Recuperado de https://bit.ly/37r2YWZ.

Ministerio de Igualdad (2020). Guía de actuación para mujeres que estén sufriendo violencia de género en situación de permanencia domiciliaria derivada del estado de alarma por COVID 19. Recuperado de https://bit. ly/37rXCuF.

Nogueiras, B. et al. (2005). "Los talleres y grupos de reflexión entre mujeres como prevención de la violencia: Una experiencia en una Concejalía de Mujer". Cuadernos de Trabajo Social, 18, pp. 351-360. Recuperado de https://bit.ly/39wTwEh.

ONU Mujeres (2020a). COVID-19 en América Latina y el Caribe: cómo incorporar a las mujeres y la igualdad de género en la gestión de la respuesta a la crisis. Recuperado de https://bit.ly/2L2dg8z.

ONU Mujeres (2020b). Policy Brief: The Impact of COVID-19 on Women. Recuperado de https://bit.ly/3qm92sA.

ONU Mujeres (6 abril, 2020). Violencia contra las mujeres: la pandemia en la sombra. Recuperado de https://bit. ly/3oh29H1.

Palacios, A. (2008). El modelo social de discapacidad: orígenes, caracterización y plasmación en la Convención Internacional sobre los Derechos de las Personas con Discapacidad. Colección CERMI № 36. Madrid: Ediciones Cinca. Recuperado de https://bit.ly/3qfzRhY.

Peláez, A. (25 marzo, 2020). "Las mujeres con discapacidad y el coronavirus" [Artículo publicado en cermi.es semanal No384]. Recuperado de https://bit.ly/3IE5oH6.

Ruiz-Pérez, I. y Pastor-Moreno, G. (2020). "Medidas de contención de la violencia de género durante la pandemia de COVID-19". Gaceta Sanitaria. Recuperado de https://bit.ly/3fYWqCM.

UNFPA (2019). Visibilizar, incluir, participar estrategia VIP. Orientaciones para promover los derechos de las personas con discapacidad en el trabajo del Fondo de Población de las Naciones Unidas en América Latina y el Caribe 2019-2021. Recuperado de https://bit.ly/3lxq3wb.

Valenciano, E. (2004). Informe sobre la situación de las mujeres de los grupos minoritarios en la Unión Europea. Recuperado de https://bit.ly/3ondXYB. 
Vicente, M. R. et al. (2005). "Una aproximación a la brecha digital por discapacidad. El caso de la Unión Europea". Boletín Económico del ICE, 2834, pp. 43-57. Recuperado de https://bit.ly/3qlmEnL.

Wenham, C. et al. (2020). "COVID-19: the gendered impacts of the outbreak". The Lancet, 395, pp. 846-848. DOI: http://dx.doi.org/10.1016/S0140-6736(20)30526-2

Women Enabled International (2020). COVID-19 at the Intersection of Gender and Disability: Findings of a Global Human Rights Survey. Recuperado de https://bit.ly/36uhEpa. 